\title{
CYLINDRICAL SHAPES OF HELFRICH SPONTANEOUS-CURVATURE MODEL
}

\author{
VLADIMIR I. PULOV AND EDDIE J. CHAKAROV
}

\section{Communicated by Jean-Francois Ganghoffer}

\begin{abstract}
The governing equation of the Helfrich spontaneous-curvature model is the Helfrich equation. It is a coordinate free equation that describes the equilibrium shapes of biological (fluid) membranes. We make use of the conformal metric representation of the Helfrich equation and by applying the symmetry group reduction method we obtain a translationally invariant solution. Based on that solution, we derive analytic expressions for the position vector of special cylindrical equilibrium shapes. Plots of the graphs of some closed directrices of these shapes are presented.
\end{abstract}

\section{Introduction}

Biomembranes (membranes of living cells), or fluid membranes as they are generally called, consist of lipid compounds, mostly phospholipid molecules, having two parts - one or two hydrocarbon tails (the hydrophobic part) and a polar head group (the hydrophilic part). Placed in aqueous solution the phospholipid molecules spontaneously assemble to form closed lipid bilayer structure: two layers of molecules locating their hydrophilic heads to point outward in order to prevent the hydrophobic tails from direct contact with the water molecules. The lipid bilayers are the typical constituents of the semipermeable membranes of all the living cells (plant and animal) playing a dominant role for determining cell's shape.

The modern biomechanical models of the fluid membranes $[2,5,20]$ dates back to the works of Canham [3] and Helfrich [7] in the early 70s of the previous century. Their basic concept, first suggested by Canham, is to think of the membrane as of a two-dimensional fluid in which the lipid molecules are moving freely in a way that no in-plane displacements can be developed, except bending. It follows from this idea that the shape of the living cells is controlled exclusively by the bending energy associated with the curvature elasticity of the lipid bilayer.

In the so-called Helfrich spontaneous-curvature model [7], the fluid membrane is described as a two-dimensional elastic surface $\mathcal{S}$, obtained as a solution of the 
Helfrich equation

$$
\Delta_{\mathcal{S}} H+2\left(H^{2}+\mathrm{lh} H-K\right)(H-\mathrm{h})-\frac{2 \lambda H}{k}+\frac{p}{k}=0
$$

where $H$ and $K$ are, respectively, the mean and the Gaussian curvatures of $\mathcal{S}$, and $\Delta_{\mathcal{S}}$ is the Laplace-Beltrami operator on $\mathcal{S}$. The Helfrich equation (1) is an equilibrium condition for attaining the minimum of the free elastic energy of the membrane

$$
\mathcal{F}=k \int_{\mathcal{S}}(H-\mathrm{h})^{2} \mathrm{~d} S+\bar{k} \int_{\mathcal{S}} K \mathrm{~d} S
$$

where the curvature energy (energy contribution due to the curvatures $H$ and $K$ ) is taken into account through the two elastic moduli of the membrane - the bending rigidity $k$ and the Gaussian rigidity $\bar{k}$. The additional curvature parameter $\mathbb{l}$, the so called spontaneous curvature, is introduced to reflect the asymmetry between the membrane and its environment. According to the stalk model, this parameter participates also in the description of the fusion of two planar membranes [6] . The pressure difference (osmotic pressure) $p$ and the tensile stress $\lambda$ are related, respectively, to the constraints of fixed volume and area of the membrane.

We are interested in immersed surfaces in the Euclidean space that satisfy the Helfrich equation (1). The necessary and sufficient condition for the immersed surface to exist is that the coefficients of its first and second fundamental form satisfy the so called Gauss-Codazzi-Mainardi integrability equations [15]. For this reason, when looking for the equilibrium surfaces of the Helfrich model, equation (1) has to be solved together with the Gauss-Codazzi-Mainardi equations. The number of equations and the order of the derivatives being involved depend on the coordinates that have been specified. In our work, we introduce the conformal metric on $\mathcal{S}$

$$
\mathrm{d} s^{2}=4 q^{2} \varphi^{2}\left(\mathrm{~d} x^{2}+\mathrm{d} y^{2}\right)
$$

and the matrix of the second fundamental form

$$
b=\left(\begin{array}{cc}
\theta & \omega \\
\omega & 8 q^{2} \varphi(1+\mathbb{h} \varphi)-\theta
\end{array}\right)
$$

so that the Helfrich equation (1) is replaced by a system of four second order partial differential equations $[9,10,18]$ (hereinafter referred to as the Helfrich system) 


$$
\begin{aligned}
& q^{2}\left(\varphi_{x x}+\varphi_{y y}\right)+2 q \varphi\left(q_{x x}+q_{y y}\right) \\
& \quad-2 \varphi\left(q_{x}^{2}+q_{y}^{2}\right)+q^{4}\left(8 \varphi+\alpha_{2} \varphi^{2}+\alpha_{3} \varphi^{3}+\alpha_{4} \varphi^{4}\right)=0 \\
& \theta_{y}-\omega_{x}-\left(8+\frac{\alpha_{2}}{3} \varphi\right) q\left(\varphi q_{y}+q \varphi_{y}\right)=0 \\
& \omega_{y}+\theta_{x}-\frac{\alpha_{2}}{3} q \varphi\left(\varphi q_{x}+q \varphi_{x}\right)-8 q \varphi q_{x}=0 \\
& 4 q^{2} \varphi\left(\varphi_{x x}+\varphi_{y y}\right)+4 q \varphi^{2}\left(q_{x x}+q_{y y}\right) \\
& \quad-4 \varphi^{2}\left(q_{x}^{2}+q_{y}^{2}\right)-4 q^{2}\left(\varphi_{x}^{2}+\varphi_{y}^{2}\right)-\omega^{2}-\theta^{2}+\left(8+\frac{\alpha_{2}}{3} \varphi\right) q^{2} \varphi \theta=0
\end{aligned}
$$

where $q=q(x, y), \varphi=\varphi(x, y), \theta=\theta(x, y)$ and $\omega=\omega(x, y)$ are unknown functions of the conformal coordinates $(x, y)$, and $\varphi_{x}=\partial \varphi / \partial x$, etc. The newly defined phenomenological constants are $\alpha_{2}=24 \mathrm{~h}, \alpha_{3}=8\left(2 \mathrm{~h}^{2}-\lambda / k\right), \alpha_{4}=$ $4 p / k-8 \lambda \mathrm{Ih} / k$. In comparison with the fourth order nonlinear partial differential equation in the Mongé representation of the Helfrich equation (see e.g. [21]), the system of differential equations (5) is a simpler version of the Helfrich model.

In the conformal metric coordinates, defined by (3) and (4), the mean $H$ and the Gaussian $K$ curvatures take the form

$$
\begin{gathered}
H=\frac{1}{\varphi}+\mathrm{h} \\
K=\frac{1}{4 q^{4} \varphi^{4}}\left(\varphi^{2}\left(q_{x}^{2}+q_{y}^{2}\right)+q^{2}\left(\varphi_{x}^{2}+\varphi_{y}^{2}\right)\right. \\
\left.-q \varphi^{2}\left(q_{x x}+q_{y y}\right)-q^{2} \varphi\left(\varphi_{x x}+\varphi_{y y}\right)\right) .
\end{gathered}
$$

The latter is obtained by using the Brioschi formula

$$
K=-\Delta \log (2 q \varphi)
$$

by making use of the respective Laplace-Beltrami operator

$$
\Delta=\frac{1}{4 q^{2} \varphi^{2}}\left(\frac{\partial^{2}}{\partial x^{2}}+\frac{\partial^{2}}{\partial y^{2}}\right)
$$

In this setting, the functional (2) reads

$$
\mathcal{F}=4 k \iint q^{2} \mathrm{~d} x \mathrm{~d} y+\bar{k} \iint \frac{8 q^{2} \varphi \theta(1+\operatorname{h} \varphi)-\theta^{2}-\omega^{2}}{4 q^{2} \varphi^{2}} \mathrm{~d} x \mathrm{~d} y
$$


and this is an obvious manifestation of the fact that the squared function $q$ plays the role of a free elastic energy density due to the mean curvature $H$ of the membrane (cf $[9,10])$.

The main goal of this paper is to apply a Lie symmetry reduction method for finding cylindrical equilibrium shapes of the Helfrich model. To this end we look for solutions of the Helfrich system with the corresponding lipid configurations having zero Gaussian curvature $K=0$. By creating and solving of the so called determining system of equations [14] we obtain the Lie symmetry algebra (respectively the Lie symmetry group) of the Helfrich system. It is shown in [10] that all the one-dimensional Lie subalgebras, of the general Lie symmetry algebra of the Helfrich system are equivalent to each other under the group of inner automorphisms. Based on this knowledge, in the next Section 2, we apply a symmetry group reduction technique [14] to obtain a group-invariant solution. In Section 4, a class of cylindrical shapes of fluid membranes are explicitly determined and some plots of closed graphs of the directrices of the obtained surfaces are presented. The solution and the directrices are expressed by the Weierstrassian functions.

\section{Group-Invariant Solution}

A group-invariant solution of a given system of partial differential equations is a solution invariant under the group action of the symmetry group of the system. The group-invariant solutions are found by solving the so called reduced system of differential equations [14]. It is of great practical importance that in comparison with the original system the reduced system of equations has fewer independent variables. Particularly, if the number of the group parameters is one less the number of the independent variables, then the initially given system of partial differential equations is reduced to a much simpler system of ordinary differential equations.

The determining system is a system of partial differential equations that is used for finding the coefficient functions $\xi^{i}(\vec{x}, \vec{u})$ and $\eta^{\alpha}(\vec{x}, \vec{u})$ of the Lie group generator $X$ of the Lie group of symmetries (Lie symmetry group) of the considered system of differential equations [14]. In the case of the Helfrich system (5) the one-parameter Lie group of symmetry transformations has the form

$$
\begin{aligned}
\tilde{x}^{i} & =\Phi^{i}(\vec{x}, \vec{u}, \varepsilon),\left.\quad \Phi^{i}\right|_{\varepsilon=0}=x^{i}, \quad i=1,2 \\
\tilde{u}^{\alpha} & =\Psi^{\alpha}(\vec{x}, \vec{u}, \varepsilon),\left.\quad \Psi^{\alpha}\right|_{\varepsilon=0}=u^{\alpha}, \quad \alpha=1,2,3,4
\end{aligned}
$$


and the Lie group generator is given by

$$
X=\sum_{i=1}^{2} \xi^{i}(\vec{x}, \vec{u}) \frac{\partial}{\partial x^{i}}+\sum_{\alpha=1}^{4} \eta^{\alpha}(\vec{x}, \vec{u}) \frac{\partial}{\partial u^{\alpha}}
$$

where $\varepsilon(\varepsilon \in I \subset \mathbb{R}, 0 \in I)$ is the group parameter and the vectors $\vec{x}=\left(x^{1}, x^{2}\right)$ and $\vec{u}=\left(u^{1}, u^{2}, u^{3}, u^{4}\right)$ denote the independent and the dependent variables, respectively: $x^{1}=x, x^{2}=y, u^{1}=q, u^{2}=\varphi, u^{3}=\theta, u^{4}=\omega$. The functions $\Phi^{i}(\vec{x}, \vec{u}, \varepsilon)$ and $\Psi^{\alpha}(\vec{x}, \vec{u}, \varepsilon)$ are found by solving the Lie equations [14]

$$
\begin{aligned}
\frac{\mathrm{d} \Phi^{i}}{\mathrm{~d} \varepsilon} & =\xi^{i}(\vec{\Phi}, \vec{\Psi}),\left.\quad \Phi^{i}\right|_{\varepsilon=0}=x^{i}, \quad i=1,2 \\
\frac{\mathrm{d} \Psi^{\alpha}}{\mathrm{d} \varepsilon} & =\eta^{\alpha}(\vec{\Phi}, \vec{\Psi}),\left.\quad \Psi^{\alpha}\right|_{\varepsilon=0}=u^{\alpha}, \quad \alpha=1,2,3,4 .
\end{aligned}
$$

The coordinates of the vectors $\vec{\Phi}$ and $\vec{\Psi}$ are $\Phi^{i}$ and $\Psi^{\alpha}$, respectively. The set of all Lie group generators constitute a Lie algebra - the Lie symmetry algebra of the Lie symmetry group.

In order to find the Lie symmetry algebra one has to solve the determining system of equations. For most of the important physical applications the determining system consists of hundreds of equations. Creating and solving of such a large system of differential equations, though algorithmically straightforward, may cause serious technical difficulties. In order to cope with the great number of tedious calculations, we take advantage of the specially developed Mathematica package LieSymm-PDE [19]. By applying the LieSymm-PDE package to the considered Helfrich system (5), we obtained a determining system of 206 first and second order partial differential equations. All these equations have the general form

$$
\sum_{k} \mu_{k}\left(u^{1}\right)^{j}\left(u^{2}\right)^{l}\left(u^{3}\right)^{m}\left(u^{4}\right)^{n} f_{k}(\vec{x}, \vec{u})=0, \quad j, l, m, n=0,1, \ldots, 7
$$

where $\mu_{k}$ are real constants and $f_{k}(\vec{x}, \vec{u})$ are either one of the functions $\xi^{i}(\vec{x}, \vec{u})$, $\eta^{\alpha}(\vec{x}, \vec{u})$ or their first or second order derivatives. Thirty five equations are with more than 10 addends (expressions of the above form), six are with more than 20 addends. The largest are two equations with 43 and 44 addends. Many of these equations are equivalent to each other or are functionally dependent, which means that the determining system is overdetermined. Nevertheless, manipulation of so many equations without making errors is quite boring and time consuming. With the aid of the LieSymm-PDE facilities for solving determining systems we managed to do all the symbolic calculations automatically, eluding the tedious 
substitutions, transformations and other technicalities, which otherwise we should had made by hand.

We started up the solving process by invoking the LieSymm-PDE iterative function for solving some predetermined types of equations with known solutions. If LieSymm-PDE identifies such an equation, its solution is substituted for the respective variable in the remainder part of the equations. In this way the determining system of the Helfrich model has been reduced to 29 partial differential equations for six unknown functions of the form

$$
\begin{array}{lll}
\xi^{1}=h(x, y), & \eta^{1}=v(x, y, q, \varphi), & \eta^{3}=g(x, y, q, \varphi, \theta, \omega) \\
\xi^{2}=r(x, y), & \eta^{2}=w(x, y, q, \varphi), & \eta^{4}=\rho(x, y, q, \varphi, \theta, \omega)
\end{array}
$$

where $h(x, y)$ and $r(x, y)$ satisfy the Cauchy-Riemann conditions

$$
\frac{\partial h}{\partial y}=-\frac{\partial r}{\partial x}, \quad \frac{\partial h}{\partial x}=\frac{\partial r}{\partial y} .
$$

We proceeded with applying the LieSymm-PDE package in an interactive mode feeding back the program with the solutions we had found. After seven interactive cycles two of the coefficient functions changed their form to

$$
\eta^{1}=q \sigma(x, y), \quad \eta^{2}=-C \varphi
$$

$(C \in \mathbb{R})$ and the determining system reduced to the 10 equations below

$$
\begin{aligned}
q \varphi\left(24+\alpha_{2} \varphi\right) \rho_{\theta}+3 \rho_{q} & =0 \\
24 q^{2} r_{x}+\alpha_{2} q^{2} \varphi \rho_{\theta}+3 \rho_{\varphi} & =0 \\
q^{2} \varphi\left(24+\alpha_{2} \varphi\right) \sigma_{x}-3 g_{x}-3 \rho_{y} & =0 \\
q^{2} \varphi\left(24+\alpha_{2} \varphi\right) \sigma_{y}-3 g_{y}+3 \rho_{x} & =0 \\
24 q^{2} r_{x}-q^{2}\left(24+\alpha_{2} \varphi\right) \rho_{\theta}-3 \rho_{\varphi} & =0 \\
3 g_{\varphi}+\alpha_{2} q^{2} \varphi \rho_{\omega}-2 \alpha_{2} q^{2} \varphi \sigma+2 \alpha_{2} C q^{2} \varphi & =0 \\
3 g_{\varphi}+q^{2}\left(24+\alpha_{2} \varphi\right) \rho_{\omega}-2 q^{2}\left(24+\alpha_{2} \varphi\right) \sigma+2 C q^{2}\left(12+\alpha_{2} \varphi\right) & =0 \\
3 g_{q}+q \varphi\left(24+\alpha_{2} \varphi\right) \rho_{\omega}-2 q \varphi\left(24+\alpha_{2} \varphi\right) \sigma+2 C q \varphi\left(12+\alpha_{2} \varphi\right) & =0 \\
2 \sigma_{x x}+2 \sigma_{y y}+2 q^{2}\left(8+\alpha_{2} \varphi+\alpha_{3} \varphi^{2}+\alpha_{4} \varphi^{3}\right) r_{y} & \\
+2 q^{2}\left(8+\alpha_{2} \varphi+\alpha_{3} \varphi^{2}+\alpha_{4} \varphi^{3}\right) \sigma-C q^{2} \varphi\left(\alpha_{2}+2 \alpha_{3} \varphi+3 \alpha_{4} \varphi^{2}\right) & =0 \\
2\left(\alpha_{2} q^{2} \varphi^{2} \theta-6 \alpha_{2} q^{4} \varphi^{3}-6 \alpha_{3} q^{4} \varphi^{4}-6 \alpha_{4} q^{4} \varphi^{5}-48 q^{4} \varphi^{2}\right. & \\
\left.+24 q^{2} \varphi \theta-3 \theta^{2}-3 \omega^{2}\right) r_{y}+\left(\alpha_{2} q^{2} \varphi^{2}+24 q^{2} \varphi-6 \theta\right) g-6 \omega \rho & \\
-6\left(2 \alpha_{2} q^{4} \varphi^{3}+2 \alpha_{3} q^{4} \varphi^{4}+2 \alpha_{4} q^{4} \varphi^{5}+16 q^{4} \varphi^{2}-\theta^{2}-\omega^{2}\right) \sigma & \\
+6 C q^{4} \varphi^{3}\left(\alpha_{2}+2 \alpha_{3} \varphi+3 \alpha_{4} \varphi^{2}\right)+6 C\left(4 q^{2} \varphi \theta-\theta^{2}-\omega^{2}\right) & =0 .
\end{aligned}
$$


Continuing in the same manner of solving we have obtained the solution of the determining system, arriving at the Lie symmetry algebra of the Helfrich system for the two distinguished cases (compare with [10])

Case 1. $\left|\alpha_{2}\right|+\left|\alpha_{3}\right|+\left|\alpha_{4}\right| \neq 0$

$$
\begin{aligned}
X^{\mathrm{I}}\left(\xi^{1}, \xi^{2}\right)=\xi^{1} \partial_{x}+\xi^{2} \partial_{y}-q \xi_{x}^{1} \partial_{q}-2\left(\theta \xi_{x}^{1}+\omega \xi_{x}^{2}\right) \partial_{\theta} & \\
& -2\left(\omega \xi_{x}^{1}-\left(\theta-4 q^{2} \varphi-\frac{\alpha_{2} q^{2} \varphi^{2}}{6}\right) \xi_{x}^{2}\right) \partial_{\omega}
\end{aligned}
$$

Case 2. $\alpha_{2}=\alpha_{3}=\alpha_{4}=0$

$$
\begin{aligned}
X^{\mathrm{II}}\left(\xi^{1}, \xi^{2}\right)= & X_{1}\left(\xi^{1}, \xi^{2}\right)+c X_{2}, \quad c \in \mathbb{R} \\
X_{1}\left(\xi^{1}, \xi^{2}\right)= & \xi^{1} \partial_{x}+\xi^{2} \partial_{y}-q \xi_{x}^{1} \partial_{q} \\
& -2\left(\theta \xi_{x}^{1}+\omega \xi_{x}^{2}\right) \partial_{\theta}-2\left(\omega \xi_{x}^{1}-\left(\theta-4 q^{2} \varphi\right) \xi_{x}^{2}\right) \partial_{\omega} \\
X_{2}= & \varphi \partial_{\varphi}+\theta \partial_{\theta}+\omega \partial_{\omega}
\end{aligned}
$$

where $\xi^{1}=h(x, y), \xi^{2}=r(x, y)$ are arbitrary real-valued harmonic functions satisfying the Cauchy-Riemann conditions (8) and $\partial_{x} \equiv \partial / \partial x$, etc.

The full sets of group generators $X^{\mathrm{I}}\left(\xi^{1}, \xi^{2}\right)$ and $X^{\mathrm{II}}\left(\xi^{1}, \xi^{2}\right)$ constitute two symmetry Lie algebra $L^{\mathrm{I}}$ and $L^{\mathrm{II}}$ for each one of the considered cases. The Lie algebras $L^{\mathrm{I}}$ and $L^{\mathrm{II}}$ are infinite dimensional with the commutator operator defined by

$$
\left[X\left(\xi^{1}, \xi^{2}\right), X\left(\widehat{\xi}^{1}, \widehat{\xi}^{2}\right)\right]=X\left(\Xi^{1}, \Xi^{2}\right)
$$

where

$$
\Xi^{1}=\xi^{1} \widehat{\xi}_{x}^{1}-\xi^{2} \widehat{\xi}_{x}^{2}-\widehat{\xi}^{1} \xi_{x}^{1}+\widehat{\xi}^{2} \xi_{x}^{2}, \quad \Xi^{2}=\xi^{2} \widehat{\xi}_{x}^{1}+\xi^{1} \widehat{\xi}_{x}^{2}-\widehat{\xi}^{2} \xi_{x}^{1}-\widehat{\xi}^{1} \xi_{x}^{2}
$$

( $X$ equals $X^{\mathrm{I}}$ or $X^{\mathrm{II}}$ respectively).

Once we have the symmetries of the Helfrich system (5), we can look for solutions that are invariant under certain subgroups of the general symmetry group. As it is shown in [10], all the group-invariant solutions of the Helfrich system, related to the one-parameter Lie symmetry subgroups of the general Lie symmetry group, are equivalent (conjugate) to each other in a sense that the related subalgebras are pairwise conjugate under the group of the adjoint representations [14]. Hence, it suffices to consider one representative of the conjugacy class of the one-parameter group-invariant solutions from which every other such solution can be derived by acting with a transformation of the symmetry group. 
We look for solutions of the Helfrich system (5) invariant under the subgroup of translations of the variable $x$, i.e., a subgroup related to the subalgebra spanned by the group generator $X^{\mathrm{I}}(1,0) \equiv \partial_{x}$. The reduction procedure leads to groupinvariant solutions of the form [10]

$$
q=q(y), \quad \varphi=\varphi(y), \quad \theta=\theta(y), \quad \omega(y)=\alpha_{5} \equiv \text { const }
$$

and the reduced system of ordinary differential equations

$$
\begin{aligned}
q^{2} \varphi_{y y}+2 q \varphi q_{y y}-2 \varphi q_{y}^{2}+q^{4}\left(8 \varphi+\alpha_{2} \varphi^{2}+\alpha_{3} \varphi^{3}+\alpha_{4} \varphi^{4}\right) & =0 \\
\theta_{y}-q\left(8+\frac{\alpha_{2}}{3} \varphi\right)\left(\varphi q_{y}+q \varphi_{y}\right) & =0 \\
4 q \varphi^{2} q_{y y}+4 \varphi q^{2} \varphi_{y y}-4 \varphi^{2} q_{y}^{2}-4 q^{2} \varphi_{y}^{2}+q^{2} \varphi \theta\left(8+\frac{\alpha_{2}}{3} \varphi\right)-\theta^{2}-\alpha_{5}^{2} & =0 .
\end{aligned}
$$

Searching for cylindrical equilibrium shapes of the Helfrich model, we confine to the case of vanishing Gaussian curvature

$$
K=0 .
$$

From (7) it follows the equation

$$
\varphi^{2} q_{y}^{2}+q^{2} \varphi_{y}^{2}=q \varphi\left(\varphi q_{y y}+q \varphi_{y y}\right)
$$

whence, by adding to both sides the expression $2 q \varphi q_{y} \varphi_{y}$, we conclude that the two functions $q(y)$ and $\varphi(y)$ must satisfy the relation

$$
q(y)=\frac{b \mathrm{e}^{c y}}{\varphi(y)}, \quad b \in \mathbb{R} / 0, \quad c \in \mathbb{R} .
$$

By substituting of this latter result in the reduced system of equations (9) it is easily observed that the solutions obtained for $c \neq 0$ do not concern the geometry of the membrane (see the explanation below).

In the following we will assume that $c=0$. Then the relation (10) takes the form

$$
q(y)=\frac{b}{\varphi(y)}, \quad b \in \mathbb{R} / 0
$$

and the system (9) is transformed to

$$
\theta(y)=0, \quad \omega(y)=0
$$

and

$$
\varphi_{y}=b\left(C_{1} \varphi^{4}-2 \alpha_{4} \varphi^{3}-\alpha_{3} \varphi^{2}-\frac{2}{3} \alpha_{2} \varphi-4\right)^{\frac{1}{2}}
$$


where $C_{1}$ is an integration constant. The obtained equation is directly solvable in terms of elliptic functions [1] (and in some special cases in elementary functions) which means that we have arrived at a group-invariant solution of the Helfrich system. Before giving any explicit solution of (12), we will introduce new variables that will reproduce the original geometrical considerations.

As it is known from the classical differential geometry [15], the directrices of the cylindrical surfaces are plane curves $\Gamma$ (Fig. 1) whose curvature $\kappa(s)$ is related to the mean curvature of the surface $H(s)$

$$
\kappa(s)=2 H(s)
$$

where the parameter $s$ is the arclength of $\Gamma$. For solutions invariant under the subgroup of translations of the variable $x$, the arclength $s$ is connected through the formula (3) to the conformal coordinate $y$

$$
s=2 y \text {. }
$$

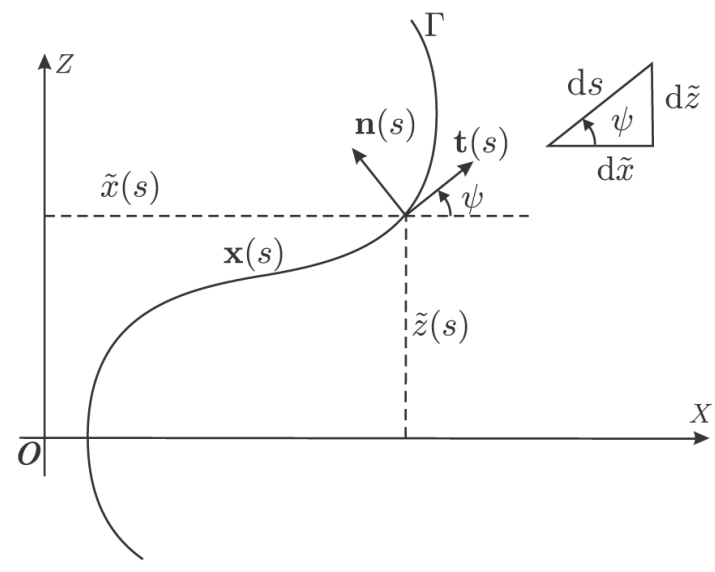

Figure 1. Geometry of a plane curve.

By changing the variables in (12) from $(y, \varphi(y))$ to $(s, \kappa(s))$, via the expression (6) for $H(y)$, a differential equation for $\kappa(s)$ is obtained

$$
\dot{\kappa}=b\left(-\frac{1}{4} \kappa^{4}+\frac{\mu}{2} \kappa^{2}+\nu \kappa+2 E\right)^{\frac{1}{2}}
$$

where the coefficients $\mu, \nu$ and $E$ are defined by

$$
\mu=4\left(\mathrm{~h}^{2}+\frac{\lambda}{k}\right), \quad \nu=-\frac{4 p}{k}, \quad E=\frac{4 \mathrm{~h}}{k}(p-\lambda \mathrm{Ih})-2 \mathbb{h}^{4}+\frac{C_{1}}{2} .
$$


Here and henceforth the dots denote the derivatives with respect to $s$. On solving the equation (13) we will first assume that the quartic polynomial

$$
f(t)=-\frac{1}{4} t^{4}+\frac{\mu}{2} t^{2}+\nu t+2 E
$$

has no repeated factors, which means that we can make use of the Weierstrassian integral form of this equation (see e.g. [23])

$$
\int_{z}^{\infty}\left(4 t^{3}-g_{2} t-g_{3}\right)^{-\frac{1}{2}} \mathrm{~d} t=b s+C_{2}
$$

where

$$
g_{2}=\frac{1}{48}\left(\mu^{2}-24 E\right), \quad g_{3}=\frac{1}{64}\left(\nu^{2}-\frac{\mu^{3}}{27}-\frac{8 \mu E}{3}\right)
$$

are the invariants of the polynomial $f(t)$. The lower limit of the integral is

$$
z=\frac{f^{\prime}(a)}{4(\kappa-a)}+\frac{f^{\prime \prime}(a)}{24}
$$

where $a$ is an arbitrary root of the polynomial and $f^{\prime}(a) \equiv \mathrm{d} f(t) / \mathrm{d} t_{\left.\right|_{t=a}}$, etc. The equation (14) is equivalent to the relation $[1,23]$

$$
z=\wp\left(b s+C_{2} ; g_{2}, g_{3}\right)
$$

where $\wp\left(b s+C_{2} ; g_{2}, g_{3}\right)$ is the Weierstrassian elliptic $\wp$-function. Combining the equations (15) and (16) we obtain the solution

$$
\kappa(s)=a+\frac{f^{\prime}(a)}{4}\left(\wp\left(b s+C_{2} ; g_{2}, g_{3}\right)-\frac{f^{\prime \prime}(a)}{24}\right)^{-1} .
$$

In the case when the equation $f(t)=0$ has multiple roots the solution can be expressed in terms of elementary functions. For example, given an arbitrary $\beta \in \mathbb{R}$, and on writing $\mu=3 \beta^{2}, \nu=2 \beta^{3}$ and $E=3 \beta^{4} / 8$, we have the polynomial $f(t)=(3 \beta-t)(\beta+t)^{3} / 4$ and the solution

$$
\kappa(s)=\frac{3 \beta-\beta^{3}\left(b s+C_{2}\right)^{2}}{1+\beta^{2}\left(b s+C_{2}\right)^{2}} .
$$

The directrices $\Gamma$ related to the above curvature are not closed and will not be considered as they do not correspond to membrane shapes. The reason for which we have omitted the solutions of the general form (10) when $c$ is not zero, is the same - they all lead to directrices of curvature $\kappa(s) \equiv 0$. Closed directrices are generated by the solution (17) - several graphs of these directrices with and without intersections are given in the next section. 


\section{Cylindrical Equilibrium Shapes}

Given the intrinsic equation $\kappa=\kappa(s)$ of a plane curve, the coordinates of its position vector $\mathbf{x}(s)=(\tilde{x}(s), \tilde{z}(s))$ are recovered in a standard way (up to a rigid motion) by calculating the quadratures [15]

$$
\tilde{x}(s)=\int_{0}^{s} \cos \psi(\tau) \mathrm{d} \tau, \quad \tilde{z}(s)=\int_{0}^{s} \sin \psi(\tau) \mathrm{d} \tau
$$

where $\psi(s)$ is the slope angle of $\Gamma$ (Fig. 1) expressed by

$$
\psi(s)=\int_{0}^{s} \kappa(\tau) \mathrm{d} \tau .
$$

For the directrices of the Helfrich cylindrical surfaces with the intrinsic equation (17) these coordinates, for $\nu \neq 0$, are given by

$$
\begin{aligned}
& \tilde{x}(s)=\frac{2}{b \nu} \dot{\kappa}(s) \cos \psi(s)+\frac{1}{\nu}\left(\kappa^{2}(s)-\mu\right) \sin \psi(s) \\
& \tilde{z}(s)=\frac{2}{b \nu} \dot{\kappa}(s) \sin \psi(s)-\frac{1}{\nu}\left(\kappa^{2}(s)-\mu\right) \cos \psi(s) .
\end{aligned}
$$

In the derivation of these formulas the integration of (18) can be avoided, which is a direct consequence of the fact that the curvature function (17) satisfies the equation (for detailed explanation cf [22] and the references cited therein)

$$
2 \ddot{\kappa}(s)+b^{2} \kappa^{3}(s)-b^{2} \mu \kappa(s)-b^{2} \nu=0 .
$$

The equation is obtained via the quadrature (19) and by making use of the integral $[1,8]$

$$
\int \frac{\mathrm{d} \tau}{\wp(\tau)-\wp(\stackrel{\circ}{\tau})}=\frac{1}{\wp^{\prime}(\stackrel{\circ}{\tau})}\left(2 \zeta(\stackrel{\circ}{\tau}) \tau+\ln \frac{\sigma(\tau-\stackrel{\circ}{\tau})}{\sigma(\tau+\stackrel{\circ}{\tau})}\right)
$$

the slope angle $\psi(s)$ in (20) is expressed in terms of the Weierstrassian functions $\wp(s), \sigma(s)$ and $\zeta(s)$

$$
\psi(s)=a b s+\frac{f^{\prime}(a)}{2 \wp^{\prime}(\stackrel{\circ}{s})}\left(2 b s \zeta(\stackrel{\circ}{s})+\ln \frac{\sigma(b s-\stackrel{\circ}{s})}{\sigma(b s+\stackrel{\circ}{s})}\right)
$$

where $\stackrel{\circ}{s}$ is defined by $\wp(\stackrel{\circ}{s})=\frac{f^{\prime \prime}(a)}{24}$. 
We are interested in the directrices $\Gamma$ that close up smoothly in the sense that there exists a real number $L$, such that

$$
\mathbf{x}(L)=\mathbf{x}(0), \quad \mathbf{t}(L)=\mathbf{t}(0)
$$

where $\mathbf{t}(s)=(\dot{\tilde{x}}, \dot{\tilde{z}})$ is the tangent vector to the curve (Fig. 1). The second one of the above two equalities, on choosing $\psi(0)=0$, implies

$$
\psi(L)= \pm 2 \pi m, \quad m=1,2,3, \ldots
$$

On the basis of the first of the equalities (23) and the formulas (20), it follows, via the equation (21), that $L$ is a multiple for some integer $n>0$ of the least period $T$ of the function $\kappa(s)$

$$
L= \pm n T \text {. }
$$

The closure condition (in the case of $\nu \neq 0$ )

$$
\psi(T)= \pm \frac{2 \pi m}{n}, \quad m, n=1,2,3, \ldots
$$

is obtained by combining (24) and (25) and the relation

$$
\psi(n T)=n \psi(T) .
$$

The latter is a direct consequence of the integral (19). The sign in (26) changes on reversing the direction of revolving the curve: $\psi(-T)=-\psi(T)$. More details about the closure conditions of the directrices of the Helfrich cylindrical surfaces can be found in [22].

Taken with respect to the moving reference frame $(\mathbf{t}(s), \mathbf{n}(s))$, built up by the tangent $\mathbf{t}(s)$ and the normal $\mathbf{n}(s)=(-\dot{\tilde{z}}, \dot{\tilde{x}})$ vectors to the curve (Fig. 1), the coordinates $(\tilde{\xi}(s), \tilde{\eta}(s))$ of the position vector

$$
\mathbf{x}(s)=\tilde{\xi}(s) \mathbf{t}(s)+\tilde{\eta}(s) \mathbf{n}(s)
$$

have the form (compare with (20))

$$
\tilde{\xi}(s)=\frac{2}{\nu b} \dot{\kappa}(s), \quad \tilde{\eta}(s)=-\frac{1}{\nu}\left(\kappa^{2}(s)-\mu\right) .
$$

Several graphs of closed directrices of the so obtained cylindrical Helfrich surfaces, defined by $(\tilde{x}, \tilde{z})$ and $(\tilde{\xi}, \tilde{\eta})$ in the respective fixed and moving reference frame, for $b=1$ and different values of the parameters $\nu, \mu, E, a$, are presented in Fig. 2 and Fig. 3.

The graphs are plotted with the help of the computer program Mathematica ${ }^{\circledR}$ exploring different values of the parameters and visualizing the curves until the 

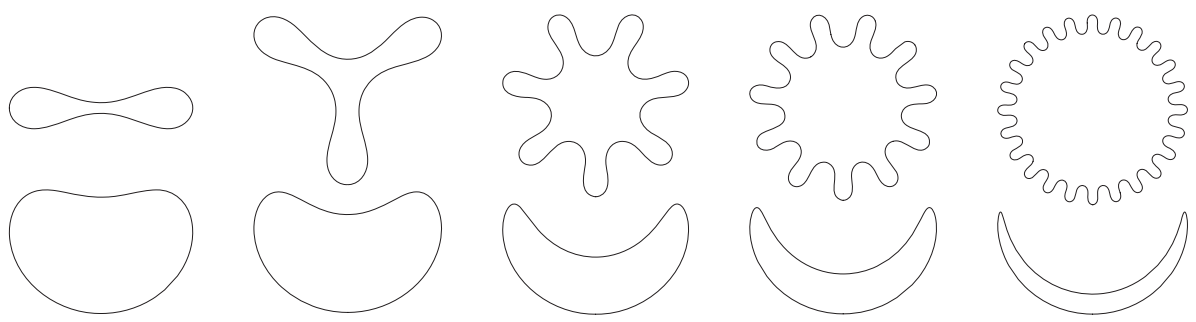

Figure 2. Closed directrices without self-intersections in the fixed (top) and the moving (bottom) reference frame.
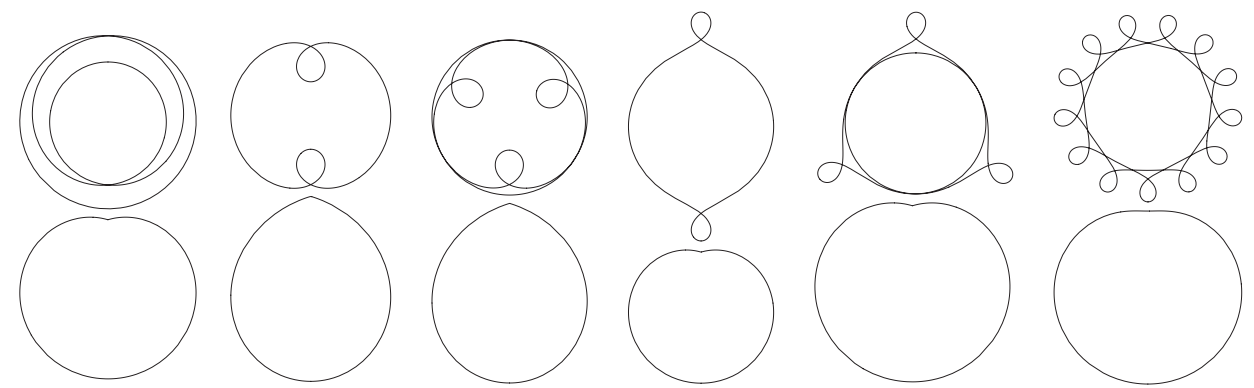

Figure 3. Closed directrices with self-intersections in the fixed (top) and the moving (bottom) reference frame.

closure condition is satisfied. The directrices on Fig. 2 have the form of rosettes with different number of ribs. As a closure condition of these curves one can make use of the empirical relation

$$
\frac{\psi(T)}{2 \pi}= \pm \frac{\gamma-1}{\gamma}, \quad \gamma=2,3, \ldots
$$

where $\gamma$ is the number of ribs. We found this formula by trying to satisfy the closure condition (26) on the base of the values of the half-periods of the Weierstrassian $\wp$-function. We fixed two of the parameters

$$
\mu=0, \quad \nu=1
$$

and then by varying the third parameter $E$, we kept calculating $T$ until an agreement with the closure condition, within the machine precision, had been reached. The directrices from left to right on Fig. 2 have been plotted with the obtained values of $E$ :

$$
0.210912, \quad 0.439387, \quad 1.24723, \quad 2.19854, \quad 6.0736 \text {. }
$$


The directrices on Fig. 3 correspond to $\mu=3, \nu=1$ and respectively, from left to right, to the parameter $E$ as follows:
0.0850036
0.0849733
0.0850046
0.0849733
0.0850046
0.034

The curves with equal number of stitches, inner and outer, have equal parameters except the root of the polynomial $a$ :

$-0.345556, \quad-2.05243, \quad-2.05245, \quad 2.74703, \quad-0.346066, \quad 2.73809$.

\section{Conclusion}

In this paper the Helfrich spontaneous curvature model has been studied from the viewpoint of the Lie groups of symmetries of the Helfrich system of equations (5). The Helfrich system is the governing system of equations for the equilibrium shapes of fluid membranes. The fluid membranes are represented here in terms of the conformal metric coordinates. The Lie group analysis of the Helfrich system has been carried out through the solution of 206 second order partial differential equations - the so called determining system of equations. The determining system has been solved in full explicit form by the help of the Mathematica ${ }^{\circledR}$ package LieSymm-PDE [19]. Its solutions constitute an infinite dimensional symmetry algebra of the Helfrich system (cf [10] where this symmetry algebra has been recently reported).

The main outcome of knowing the Lie symmetry group of a given system of differential equations is the possibility to apply a special group reduction technique, so that a reduced system of equations with fewer number of independent variables is obtained. Each solution of the reduced system remains unchanged under the group action. Such solutions are called group-invariant solutions.

We have found a translationally invariant solution that corresponds to generalized cylindrical surfaces. As it is shown in the aforementioned paper [10] all the oneparameter group-invariant solutions of the Helfrich system can be obtained from this solution by acting with an appropriate group transformation. It means that the solutions of the Helfrich system (5) that are invariant with respect to one-parameter subgroups of the general symmetry group are equivalent to each other.

We expressed the obtained solution in terms of the Weierstrassian functions. In the case of $\nu \neq 0$, we provide explicit expressions for the position vector of the directrices of the cylindrical surfaces by the equations (17), (20) and (22). With the help of Mathematica ${ }^{\circledR}$ we have explored different values of the free parameters in the Helfrich system in order to obtain smoothly closing directrices. 
Most of the graphs of the directrices that we present here (Fig. 2 and Fig. 3) have similar shapes to the plots of the graphs in reference [22], which is not so much surprising as the graphs in [22] also represent the directrices of cylindrical Helfrich surfaces, but note that they are plots of solutions obtained by another approach that lead to Jacoby and not to the Weierstrassian elliptic functions. The similarity of the graphs shows that their analytic expressions are different representations of one and the same solution to the Helfrich equation (1). As a matter of future work it is a challenging perspective to get more inside in this similarity and the mathematical relations behind it. It is also a good problem to reveal how the choice of the root $a$ of the polynomial involved impacts the curves as those on Fig. 3.

Solutions of the Helfrich equation (1) that have been recovered in [10] from the group invariant solutions of the Helfrich system (5) corresponding to equilibrium membrane shapes known in the literature: sphere [17], Clifford torus [16], circular cylinder [17], Delaunay surfaces [11,13] and circular biconcave discoid $[11,12]$. They have been obtained on the basis of several simplifying assumptions for the four unknown functions $q(y), \varphi(y), \theta(y)$ and $\omega(y)$ and specific relations between them. Here, by assuming that $q(y)$ and $\varphi(y)$ satisfy the equation (11), this list of Helfrich surfaces obtained from the Lie group method for finding group-invariant solutions have been extended with the generalized cylindrical Helfrich shapes [22]. Substitutions that will reduce the system (9) to quadratures describing other classes of surfaces (e.g. the generalized DelaunayŠs unduloids [4]) can also be envisaged as a future work.

\section{Acknowledgements}

The first named author acknowledges the financial support provided by the European Social Fund and the Ministry of Education and Science of Bulgaria under the project BG051PO001-3.3.05-0001 "Science and Business" of the Operational Programme "Human Resources Development".

The authors owe special thanks to Professor Ivaïlo Mladenov for many inspiring discussions on the geometry of membranes.

\section{References}

[1] Abramowitz M. and Stegun I. (Eds), Handbook of Mathematical Functions, Dover, New York 1972.

[2] Brown F., Elastic Modeling of Biomembranes and Lipid Bilayers, Annu. Rev. Phys. Chem. 59 (2008) 685-712. 
[3] Canham P., The Minimum Energy of Bending as a Possible Explanation of the Biconcave Shape of the Human Red lood Cell, J. Theor. Biol. 26 (1970) 61-81.

[4] Djondjorov P., Hadzhilazova M., Mladenov I. and Vassilev V., Beyond Delaunay Surfaces, J. Geom. Symmetry Phys. 18 (2010) 1-11.

[5] Faller R., Jue T., Longo M. and Risbud S. (Eds), Biomembrane Frontiers: Nanostructures, Models, and the Design of Life, Handbook of Modern Biophysics vol. 2, Humana Press, New York 2009.

[6] Hadzhilazova M. and Ganghoffer J.-F., Membrane Fusion Based on the Stalk Model, Bulg. Chem. Comm. 46 (2014) 62-67

[7] Helfrich W., Elastic Properties of Lipid Bilayers: Theory and Possible Experiments, Z. Naturforsch C 28 (1973) 693-703.

[8] Janhke E., Emde F. and Lösch F., Tafeln Höherer Funktionen, Teubner, Stuttgart 1960.

[9] Konopelchenko B., On Solutions of the Shape Equation for Membranes and Strings, Phys. Lett. B 414 (1997) 58-64.

[10] de Matteis G. and Manno G., Lie Algebra Symmetry Analysis of the Helfrich and Willmore Surface Shape Equations, Comm. Pure Appl. Anal. 13 (2014) 453-481.

[11] Mladenov I., New Solutions of the Shape Equation, Eur. Phys. J. B 29 (2002) 327-330.

[12] Naito H., Okuda M. and Ou-Yang Z.-C., Counterexample to Some Shape Equations for Axisymmetric Vesicles, Phys. Rev. E. 48 (1993) 2304-2307.

[13] Naito H., Okuda M. and Ou-Yang Z.-C., New Solutions of the Helfrich Variational Problem for the Shapes of Lipid Bilayer Vesicles: Beyond Delaunay Surfaces, Phys. Rev. Lett. 74 (1995) 4345-4348.

[14] Olver P., Applications of Lie Groups to Differential Equations, Springer, Berlin 1986.

[15] Oprea J., Differential Geometry and its Applications, Mathematical Association of America, Washington D. C. 2007.

[16] Ou-Yang Z.-C., Anchor Ring-Vesicle Membranes, Phys. Rev. A 41 (1990) $4517-4520$.

[17] Ou-Yang Z.-C. and Helfrich W., Bending Energy of Vesicle Membranes: General Expressions for the First, Second, and Third Variation of the Shape Energy and Applucations to Spheres and Cylinders, Phys. Rev. A 39 (1989) 5280-5288. 
[18] Pulov V., Chacarov E., Hadzhilazova M. and Mladenov I., Symmetry Properties of the Membrane Shape Equation, Geometry, Integrability \& Quantization, 14 (2013) 152-159.

[19] Pulov V., Chacarov E. and Uzunov I., A Computer Algebra Application to Determination of Lie Symmetries of Partial Differential Equations, Serdica J. Computing 1 (2007) 505-518.

[20] Tu Z.-C., Geometry of Membranes, J. Geom. Symmetry Phys. 24 (2011) 4575; arXiv:1106.2370 [cond-mat.soft].

[21] Vassilev V, Djondjorov P. and Mladenov I., Symmetry Groups, Conservation Laws and Group-Invariant Solutions of the Membrane Shape Equation, Geometry, Integrability \& Quantization 7 (2006) 265-279.

[22] Vassilev V., Djondjorov P. and Mladenov I., Cylindrical Equilibrium Shapes of Fluid Membranes, J. Phys. A: Math. \& Theor. 41 (2008) 435201, 16 pp.

[23] Whittaker E., Watson G., A Course of Modern Analysis, Reprinted from Fourth Edition, Cambridge University Press, Cambridge 1996.

Vladimir I. Pulov

Department of Physics

Technical University of Varna

Varna 9010, Bulgaria

E-mail address: vpulov@hotmail.com

Eddie J. Chakarov

Department of Informatics

Varna Free University

Varna 9000, Bulgaria

E-mail address: eddy@abv.bg 